$\underline{\text { Preprint typeset in JHEP style - HYPER VERSION }}$

May 25, 2022

\title{
Kerr-CFT From Black-Hole Thermodynamics
}

\author{
Bruno Carneiro da Cunha ${ }^{a}$, Amilcar R. de Queiroz ${ }^{b, c *}$, \\ ${ }^{a}$ Departamento de Física, Universidade Federal de Pernambuco, 53901-970, \\ Recife, Pernambuco, Brazil \\ ${ }^{b}$ Instituto de Física, Universidade de Brasília, Caixa Postal 04455, 70919-970, \\ Brasilia, DF, Brazil \\ ${ }^{c}$ International Center for Condensed Matter Physics (ICCMP), Universidade de \\ Brasília, Caixa Postal 04667, Brasília, DF, Brazil
}

\begin{abstract}
We analyze the near-horizon limit of a general black hole with two commuting killing vector fields in the limit of zero temperature. We use black hole thermodynamics methods to relate asymptotic charges of the complete spacetime to those obtained in the near-horizon limit. We then show that some diffeomorphisms do alter asymptotic charges of the full spacetime, even though they are defined in the near horizon limit and, therefore, count black hole states. We show that these conditions are essentially the same as considered in the Kerr/CFT corresponcence. From the algebra constructed from these diffeomorphisms, one can extract its central charge and then obtain the black hole entropy by use of Cardy's formula.
\end{abstract}

KEYwords: Kerr-CFT, Extremal Black Hole, Duality, Virasoro Algebra, Centra Charges.

*bcunha@df.ufpe.br, amilcarq@unb.br 


\section{Contents}

1. Introduction 1

2. Two commuting Killing fields 3

3. The Near Horizon limit

4. The Boundary Conditions 7

5. Central Charges 11

5.1 Frolov Temperature 13

5.2 Entropy

6. Conclusions 15

\section{Introduction}

Central charges of symmetry algebras are helpful to either organize the dynamics of a classical system or the spectrum of a quantum one [1]. In three dimensional gravity, a particularly relevant set of charges found by Brown and Henneaux [2] allows for an interpretation of the states of the theory as diffeomorphisms of space-time. In that particular example, the states arise as coordinate transformations which leave the asymptotic structure of $\mathrm{AdS}_{3}$ invariant but do modify the subleading terms and thus change the conserved quantities. Assuming that the underlying theory is unitary, one can then use generic results in two-dimensional conformal field theory [3] to count the number of states in the spectrum. This in turn paved way for the Strominger-Vafa calculation in string theory [4], where the same Virasoro algebra was found from the string excitations of the $D 1-D 5$ system. Then the appearance of classical central charges became an indication that one could understand the entropy of black holes, or gravitational systems in general, as somehow counting the number of diffeomorphically related but inequivalent metrics.

In Guica et al. [5], an interesting example of the procedure outlined above was put forward for the extremal Kerr black hole. The really interesting feature of the 
correspondence in that case is that it does not apparently rely on supersymmetry. Instead, the idea is based on the fact that, in the case of the extremal Kerr black hole, the near-horizon limit has as isometry the algebra $S L(2, \mathbb{R}) \times U(1)$, as remarked by Bardeen in the 70's (for a more recent application, [6]). An appropriate choice of boundary conditions of this near-horizon geometry $A d S_{2} \times S^{2}$ allow them to show that the $U(1)$ sector of the isometry algebra can be enhanced to a Virasoro algebra with central charge $c=12 \mathrm{~J} / \hbar$. From this algebra Cardy's formula was used to compute the entropy, assigning to the extremal horizon the Frolov temperature [7]. The result, $S=\pi^{2} c T / 3=2 \pi J / \hbar$, exactly reproduces the celebrated BekensteinHawking entropy of the extremal Kerr black hole.

There were many works applying the procedure to other examples of extremal spinning black holes. A partial list include: Kerr-Bolt Spacetimes [8], extremal KerrSen black hole that appears as solutions in the low energy limit of heterotic string theory in 5D [9], extremal rotating Kaluza-Klein black holes [10], D1-D5-P and the BMPV black holes $[11,12]$, general rotating black hole solutions in gauged and ungauged supergravities [13,14], and solutions of higher order theories of gravity [15]. Furthermore, by considering different boundary conditions another realization of the Virasoro algebra of the above procedure was also considered in [16,17]. A partial classification of distinct boundary conditions are discussed in [18].

As stated above, a key step in [5] is the appropriate choice of the boundary conditions. In their work, they use Barnich and Brandt [19] formulas for the central charges, and by a posteriori inspection of these formulas they were able to discover boundary conditions allowing for Virasoro algebra. Now, it is certainly desirable to be able to obtain these boundary conditions via more physical arguments.

In the present work, we give some arguments on some necessary physical conditions that leads to these boundary conditions. For that we pose the near-horizon limit in an interesting form (3.13). That allows us to see this near-horizon limit as a decoupling limit, and therefore understand it as a renormalization group flow, typical of gauge/gravity dualities, in a still-to-be-found dual gauge theory. We next apply this renormalization group flow reasoning to relate observables in the Kerr space-time to (usual infinite) near-horizon observable. In particular, we apply this to the case of the mass, obtaining thus condition 4.9. From this condition, it is straightforward to obtain the boundary conditions [5].

The present work is organized as follows: in Section 2, we present some general geometric conditions for a space-time to yield a (fibered) $A d S_{2} \times X$ geometry in the near-horizon limit; in Section 3, we use the zeroth law of black hole thermodynamics 
and the extremality condition to obtain the near-horizon limit. We then write and discuss the near-horizon limit as a decoupling limit; in Section 4, boundary conditions for the near-horizon geometry that leads to a Virasoro algebra are obtained by using the first law of black hole thermodynamics. We then argument that this boundary conditions can be obtained by relating full space-time observables to near-horizon observables; in Section 5, we obtain the central charge of these Virasoro algebra and use it to obtain the microscopic entropy of the extremal spinning black holes. We close this letter with concluding remarks.

\section{Two commuting Killing fields}

There are many examples of space-times that allow for a fibered $\mathrm{AdS}_{2} \times X$ description in the near horizon limit. In fact, the program began almost forty years ago [20]. In all examples, one starts from a regular solution of gravity with a number of symmetries and by a scaling limit, enhances the symmetry to $\mathrm{SL}(2, \mathbb{R})$, that of $\mathrm{AdS}_{2}$. The scaling limit is taken by directly choosing a set of coordinates, and although some of the coordinates have a direct geometric interpretation (like $t$ ), some do not (like $r$ ). In the spirit of general covariance, we will set geometrically the conditions under which the near-horizon limit of a black hole will yield a fibered $\mathrm{AdS}_{2} \times X$ geometry.

We start with a solution allowing two commuting Killing vector fields. A time translation $\partial / \partial \bar{t}$ will give us a definite global energy and a rotation $\partial / \partial \bar{\phi}$ will yield one combination of the angular momenta entering the first law. Both generators commute between themselves. Indeed, the action of either generator will keep invariant the conserved charge associated with the other generator. Following the discussion in Sec. 7.1 of [21], we choose coordinates $\bar{t}, \bar{\phi}, x^{i}$, such that the metric makes full use of the symmetries.

Obviously, the metric components are functions of $x^{i}$ alone. But, when (i) either Killing vector field vanishes at at least one point of your space-time and (ii) the threeform generated by the outer product of both Killing fields and the Ricci tensor applied to either of them vanishes, the metric can be put in a form where the components $g_{\bar{t} \mu}$ and $g_{\bar{\phi} \mu}$ all vanish. These conditions of Theorem 7.1.1 in [21] are met for the cases of interest, since (i) the rotation vanishes at the axis of symmetry and (ii) all solutions are supposed to be either vacuum flat or vacuum anti-de Sitter, and in either case $R_{b}^{a}$ is zero or proportional to the identity.

On these space-times, one can choose the coordinates $x^{i}$ in the surfaces orthogonal to the curves parametrized by $\bar{t}$ and $\bar{\phi}$, so that the interval element takes the 
form:

$$
d s^{2}=-V d \bar{t}^{2}+2 W d \bar{t} d \bar{\phi}+X d \bar{\phi}^{2}+g_{i j} d x^{i} d x^{j}
$$

where $V, W, X$ and $g_{i j}$ are functions of $x^{i}$. The function

$$
\rho^{2}=V X+W^{2}
$$

parametrizes the volume element of the $\bar{t}-\bar{\phi}$ plane and its vanishing is signal that at least one linear combination of the Killing vector field has vanishing norm, $i$. e., signals the existence of a Killing horizon, which appears in all known black hole solutions which display an event horizon. We will assume that $\nabla_{a} \rho \neq 0$, and, for now, the rest of the coordinates $x^{i}$ constant along the integral curves of $\nabla^{a} \rho$, so that $g_{\rho i}=0$. The line element for a generic space-time allowing for two commuting Killing vector fields is then

$$
d s^{2}=-\frac{\rho^{2}}{X} d \bar{t}^{2}+X(d \bar{\phi}-\omega d \bar{t})^{2}+g_{\rho \rho} d \rho^{2}+g_{i j} d x^{i} d x^{j},
$$

where $\omega=-W / X$.

This form of the metric above reduce to a variety of metrics studied in the KerrCFT correspondence. For instance, the Kerr solution in Newman coordinates are obtained for

$$
\begin{gathered}
\rho^{2}=\Delta \sin ^{2} \theta, \quad X=\sin ^{2} \theta \frac{\left(\tilde{r}^{2}+a^{2}\right)^{2}-\Delta a^{2} \sin ^{2} \theta}{\tilde{r}^{2}+a^{2} \cos ^{2} \theta}, \\
\omega=\frac{2 M \tilde{r} a}{\Delta\left(\tilde{r}^{2}+a^{2} \cos ^{2} \theta\right)} \quad \Delta=r^{2}-2 M r+a^{2} .
\end{gathered}
$$

Note that the horizon is situated at $\Delta=0$ and that the transverse coordinate $\theta$ differs from the usual Kerr solution by a $\rho$-dependent term, so that we have $g_{\rho \theta}=0$, rather than $g_{\tilde{r} \theta}=0$.

\section{The Near Horizon limit}

We will now study (2.3) close to the horizon $\rho^{2}=0$. In the general case, the functions $X, \omega$ all have finite limits there. They define respectively the radius of the orbits of $\partial / \partial \phi$ and the angular velocity on the horizon. The component $g_{\rho \rho}$ merits some extra attention: it is the norm of the vector $\partial / \partial \rho$, defined to be the conjugate vector to the gradient $(d \rho)_{a}=\nabla_{a} \rho$. Therefore $\left(\nabla^{a} \rho\right)\left(\nabla_{a} \rho\right)=\left(g_{\rho \rho}\right)^{-1}$. Define $\chi^{a}=(\partial / \partial t)^{a}-\omega(\partial / \partial \phi)^{a}$ the Killing vector field which does vanish at the 
horizon $\rho^{2}=0$. The zeroth law of black hole thermodynamics state that the function

$$
\kappa^{2}=-\frac{1}{4} \frac{\nabla_{a}\left(\chi^{b} \chi_{b}\right) \nabla^{a}\left(\chi_{c} \chi^{c}\right)}{\chi^{d} \chi_{d}}
$$

is constant at the horizon and there it defines the surface gravity for static (stationary) black holes. Note also that $\omega$ is constant at $\rho^{2}=0$ and likewise defines the angular velocity $\Omega_{H}$ of the horizon. Using the functions $V, W$ and $X$, we can write $\chi_{a} \chi^{a}$ as

$$
\chi^{a} \chi_{a}=-V-2 \frac{W}{X} W+\frac{W^{2}}{X^{2}} X=-\frac{\rho^{2}}{X} .
$$

The definition of $\kappa$ will result on the following expression for $g_{\rho \rho}$,

$$
\left(g_{\rho \rho}\right)^{-1}=X \kappa^{2}-\frac{\rho^{2}}{2 X^{2}} \nabla_{a} X \nabla^{a} X+\frac{\rho}{X^{2}} \nabla_{a} \rho \nabla^{a} X
$$

The expression (3.3) for $g_{\rho \rho}$ above shows that, if the horizon has finite surface gravity, the scaling limit of the metric (2.3) will show nothing out of the ordinary. If, however, $\kappa \rightarrow 0$, the black hole becomes extremal and $g_{\rho \rho}$ diverges at small $\rho$ like $\mathcal{O}\left(\rho^{-2}\right)$. Note that, assuming that $X$ is finite at $\rho=0$, the last term in (3.3) also gives a contribution of $\mathcal{O}\left(\rho^{2}\right)$ to $\left(g_{\rho \rho}\right)^{-1}$. We will then assume that

$$
g_{\rho \rho}=\frac{A}{\rho^{2}}+\mathscr{O}\left(\rho^{0}\right), \quad \omega=\Omega_{H}+\bar{\Omega} \rho+\mathscr{O}\left(\rho^{2}\right),
$$

for $A$ and $\bar{\Omega}$ functions of $x^{i}$.

The dependence of $A$ in the transverse coordinates can be removed by a redefinition of $\rho$ and $x^{i}$. Using the liberty to redefine $\rho$ by a function of $x^{i}$, one can make

$$
\rho \rightarrow \Phi \rho, \quad x^{i} \rightarrow x^{i}+A^{i}
$$

such that

$$
g_{i j} \frac{\partial A^{i}}{\partial \rho} \frac{\partial A^{j}}{\partial \rho}=\frac{\rho^{2}}{X}\left(\Phi^{2}-A\right) \quad \text { and } \quad g_{i j} \frac{\partial A^{i}}{\partial \rho} \frac{\partial A^{j}}{\partial x^{k}}=-\frac{A}{X \Phi \rho} \frac{\partial \Phi}{\partial x^{k}} .
$$

The result is a metric of the form

$$
d s^{2}=\frac{\Phi^{2}}{X}\left(-\rho^{2} d \bar{t}^{2}+\frac{d \rho^{2}}{\rho^{2}}\right)+X(d \bar{\phi}-\omega d \bar{t})^{2}+\bar{g}_{i j} d x^{i} d x^{j}+\ldots
$$


where the ellipsis include the subleading terms of $g_{\rho \rho}$. Taking the scaling limit

$$
\bar{t}=\lambda^{-1} t, \quad \rho=\lambda r, \quad \bar{\phi}=\phi-\Omega_{H} \lambda^{-1} t, \quad \lambda \rightarrow 0
$$

of this metric will yield one of the type $\mathrm{AdS}_{2} \times X$

$$
d s_{\text {nhe }}^{2}=\Omega^{2}\left(-r^{2} d t^{2}+\frac{d r^{2}}{r^{2}}+\Lambda^{2}(d \phi-r d t)^{2}\right)+\tilde{g}_{i j} d x^{i} d x^{j}
$$

with the subscript "nhe" stands for "near-horizon limit of the extremal black hole", and where $\Omega, \Lambda$ and $\tilde{g}_{i j}$ are computed at the horizon and, although may depend on the other angular variables $x^{i}$, do not of course depend on $r$ anymore. Furthermore, $r_{0}$ is now a constant.

The near horizon metric (3.9) displays two new symmetries. One symmetry is

$$
r \rightarrow c r, \quad t \rightarrow \frac{t}{c}
$$

generated by the vector field

$$
\xi_{3}^{a}=r\left(\frac{\partial}{\partial r}\right)^{a}-t\left(\frac{\partial}{\partial t}\right)^{a} .
$$

Another symmetry is generated by the vector field

$$
\xi_{+}^{a}=\left(r^{-2}+t^{2}\right)\left(\frac{\partial}{\partial t}\right)^{a}-2 r t\left(\frac{\partial}{\partial r}\right)^{a}-\frac{\beta}{r}\left(\frac{\partial}{\partial \phi}\right)^{a},
$$

with $\beta$ a constant. These vector fields will, along with $\psi^{a}=(\partial / \partial \phi)^{a}$ and $\xi_{-}^{a}=$ $(\partial / \partial t)^{a}$ form a full $\mathrm{SL}(2, \mathbb{R}) \times \mathrm{U}(1)$ symmetry.

The role of the scaling transformation (3.8) is to decouple the degrees of freedom at the horizon to the ones external to the Black Hole region. At first this effect may seem at odds with the fact that (3.8) is a change of coordinates, and hence a "pure gauge" transformation. Schematically, the transformation can be cast as

$$
g_{a b}^{\text {nhe }}=\lim _{\beta \rightarrow \infty} \exp \left(-\beta \mathscr{L}_{\xi_{3}}\right) \hat{K} g_{a b},
$$

where the effect of $\hat{K}$ is to take $\bar{\phi}+\Omega_{H} \bar{t}$ to $\phi$, the affine parameter to the Killing vector field that becomes null at the horizon. This transformation changes dramatically the asymptotic characteristics of the metric, which come from being asymptotically flat to asymptotically anti-de Sitter. Therefore it cannot be considered a true diffeo- 
morphism. We will have more to say along these lines in the next section.

From the definition above it is also obvious that the dilation operator $\xi_{3}$ will be a symmetry of $g_{a b}^{\text {nhe }}$. That leads to the question of why the procedure doesn't work with metrics away from extremality, like, for instance, Schwarzschild. The answer is that, when the temperature of the horizon is not zero, the limit simply does not exist. Indeed, as one inspects some components of the metric, like (3.3), one sees that, unless $\kappa=0$, the relevant term of the interval element blows up in the limit $\lambda \rightarrow \infty$.

Decoupling limits like (3.8) are at the heart of the gauge-gravity duality, and the holographic view of the global symmetries relates scale transformations to the renormalization group flow. Thus, the appearance of a dilation symmetry at the horizon points to the fact that we are dealing with an infrared fixed point of the dual theory. It would be interesting to find explicit examples of such dual theories and their infrared fixed point counterparts.

\section{The Boundary Conditions}

As we saw in the last section, the structure of the near horizon metric is universal and is a direct result of the vanishing of the temperature of a Killing horizon. As the relevant quantities are along the $t, \phi$ and $r$ direction, one can wonder whether the Brown-Henneaux technique [2] will help us count gravitational states. At the core of the technique is the notion that some coordinate changes alter the asymptotic charges, like the mass or the angular momentum, and therefore fail to be true diffeomorphisms of the solution.

For asymptotically flat solutions of theories whose gravitational sector reduces at low energy to the Einstein-Hilbert Lagrangian, these charges are given by

$$
M=-\frac{1}{8 \pi} \int_{S} \varepsilon_{a_{1} \ldots a_{n-2} b c} \nabla^{b} \xi^{c}, \text { and } J=-\frac{1}{16 \pi} \int_{S} \varepsilon_{a_{1} \ldots a_{n-2} b c} \nabla^{b} \psi^{c}
$$

where $S$ is a "sphere at infinity". The expressions above allow for proofs of the laws of the thermodynamics of black holes. By using Stokes' theorem on the Komar formula for the asymptotic mass above, one arrives at

$$
M=2 \int_{\Sigma} \xi^{e} R_{e}^{d} \epsilon_{a_{1} \ldots a_{n-1} d}-\frac{1}{8 \pi} \int_{\mathcal{H}} \varepsilon_{a_{1} \ldots a_{n-2} b c} \nabla^{b} \xi^{c}
$$

This relates the total mass of the space-time with quantities computed at the horizon of the black hole $\mathcal{H}$. Indeed, by writing the time translation Killing vector field as 
$\chi^{a}-\Omega_{H} \psi^{a}$, where $\chi^{a}$ is the Killing vector associated with the horizon, and $\psi^{a}$ the one related to rotations, one can show that this surface term is

$$
-\frac{1}{8 \pi} \int_{\mathcal{H}} \varepsilon_{a_{1} \ldots a_{n-2} b c} \nabla^{b} \xi^{c}=\frac{1}{4 \pi} \kappa A+2 \Omega_{H} J_{H}
$$

with $\Omega_{H}$ the "angular velocity" of the horizon and $J_{H}$ the angular momentum of the black hole. Given a metric variation $\delta g_{a b}=\gamma_{a b}$ satisfying the linearized equations of motion $\delta R_{a b}=0$, the change in the total mass is given by [22]:

$$
\delta M=-\frac{1}{8 \pi} \int_{S} \varepsilon_{a_{1} \ldots a_{n-2} b c} \xi^{b} \nabla_{d}\left(\gamma^{c d}-g^{c d} \gamma\right)
$$

which is essentially the variation of the bulk term of (4.2), using the usual formulas for the variation of the Ricci tensor ${ }^{1}$. In the case where the linearized equations of motion are satisfied, the domain of integration can be again brought to the horizon:

$$
\delta M=-\frac{1}{8 \pi} \int_{S} \varepsilon_{a_{1} \ldots a_{n-2} b c} \xi^{b} \nabla_{d}\left(\gamma^{c d}-g^{c d} \gamma\right)=-\frac{1}{8 \pi} \int_{\mathcal{H}} \varepsilon_{a_{1} \ldots a_{n-2} b c} \xi^{b} \nabla_{d}\left(\gamma^{c d}-g^{c d} \gamma\right)
$$

The evaluation of these quantities at the horizon are tied to the variation of the intensive quantities $\kappa$ and $\Omega_{H}$. Evaluating it [23], one arrives at the Black Hole Gibbs relation:

$$
\delta M=-\frac{1}{4 \pi} A \delta \kappa-2 J_{H} \delta \Omega_{H} .
$$

Given a metric variation resulting from the application of a diffemorphism $\gamma_{a b}=$ $2 \nabla_{(a} \eta_{b)}$, one usually expects the asymptotic charges to remain the same. In fact, these operations are the gravitational analogue of gauge transformations and as such cannot alter the value of gauge-invariant observables. However, some gauge transformations, dubbed large, do change asymptotic holonomies and hence can alter boundary conditions like the values of the charges. Perhaps the most common case of the appearance of those are in instantons in non-abelian gauge theories. In [2] the authors noticed that these also arise in three-dimensional gravity with negative cosmological constant, which can be seen as a Chern-Simons gauge theory. There, the appropriate gauge group is comprised by local isometries of the metric, $\mathrm{SL}(2, \mathbb{R}) \times \mathrm{SL}(2, \mathbb{R})$. These gauged transformations are locally diffeomorphisms, but may not satisfy boundary conditions that keep the asymptotic structure intact. As large gauge transformations change the value of observables like the total mass and angular momentum, they should be seen as global symmetries rather than spurious

\footnotetext{
${ }^{1}$ See, for instance, $(7.5 .14)$ in $[21]$.
} 
transformations usually associated with gauge invariance [24]. Being global symmetries, these large gauge transformation do fulfill the bootstrap program to be used to organize the spectrum and allow for the counting of states. In the case of Brown and Henneaux [2], the global algebra can be written in terms of two copies of Virasoro generators and, after assuming unitarity and modular invariance, character formulas [3] can be used - sucessfully - to account for the Bekenstein-Hawking formula for the black hole entropy.

One sees then that the interpretation of the entropy of the black hole as large gauge transformations is intimately tied to the choice of boundary conditions. The idea is to allow for boundary conditions to the gauge fields that allow for changing of the asymptotic charges. On the other hand, too lax a falloff condition would allow for infinite changes in those charges. This situation is also undesirable for the purpose of counting states while keeping thermodynamical quantities fixed.

In [5] a proposal for suitable boundary conditions was made, based on the variation of general formulas developed by Barnich and Brandt [19]. The boundary conditions were given in terms of the near-horizon limit coordinates (3.9) which cloud their interpretation as modifiers of the asymptotic charges of the full space. The conditions are $g_{a b}=\bar{g}_{a b}+\gamma_{a b}$ where $\bar{g}_{a b}$ is the metric associated with the interval element of "global" $\mathrm{AdS}_{2} \times X$

$$
d s^{2}=\Omega^{2}\left(-\left(1+\varrho^{2}\right) d \tau^{2}+\frac{d \varrho^{2}}{1+\varrho^{2}}+\Lambda^{2}\left(d \varphi-\frac{\varrho}{\varrho_{0}} d \tau\right)^{2}\right)+\tilde{g}_{i j} d x^{i} d x^{j}
$$

and $\gamma_{a b}$ are terms vanishing at the conformal boundary $\varrho=\infty$ like

$$
h_{\mu \nu}=\left(\begin{array}{ccc}
\mathcal{O}\left(\varrho^{2}\right) & \mathcal{O}(1) & \mathcal{O}\left(\varrho^{-2}\right) \\
h_{\phi t} & \mathcal{O}(1) & \mathcal{O}\left(\varrho^{-1}\right) \\
h_{r t} & h_{r \phi} & \mathcal{O}\left(\varrho^{-3}\right)
\end{array}\right)
$$

and terms depending on the angular variable $g_{i \mu}=\mathcal{O}\left(\varrho^{-1}\right)$ except $g_{i r}=\mathcal{O}\left(\varrho^{-2}\right)$.

These boundary conditions are rather odd from the near-horizon, i.e., the $\mathrm{AdS}_{2}$ point of view. In fact, the conditions do not even maintain the "asymptotic triviality" of the metric, which can be seen from the fact that $h_{\tau \tau}$ is of the same order in $\varrho$ as $g_{\tau \tau}$.

These boundary conditions can however be rather straightforwardly derived from the requirement that the full space modification of the asymptotic charges to be finite. The relation between charges in the full Kerr metric (2.3) and in the near 
horizon limit (3.9) is surprisingly direct. As a matter of fact, the relation between them is the scaling limit (3.8), which, for finite $\lambda$, is just a change of coordinates. Moreover, quantities like the volume element do not change under scaling, and the only perceptible difference stems from the transformation of the time coordinate. Therefore,

$$
M=\lambda M_{\lambda}^{\text {nhe }}
$$

where $M_{\lambda}^{\text {nhe }}$ refers to the near horizon geometry capped at small, but finite, $\lambda$. It is clear then, that while the metric obtained at the limit $\lambda \rightarrow 0$ is perfectly reasonable, the charges computed in this limit may be infinite and still be related to finite changes of the total mass of the Kerr space-time.

The near horizon limit is implemented at the metric level, as an expansion over $\lambda:$

$$
g_{a b}=\bar{g}_{a b}+\lambda h_{a b}+\ldots
$$

and substitution $g_{a b}=\bar{g}_{a b}$ valid insofar as the corrections $\lambda h_{a b}$ are small. This fail to be the case at large $r$, or $\varrho$. We will then consider the near-horizon geometry up to scales of order $r \propto \lambda^{-1}$, and define $M_{\lambda}^{\text {nhe }}$ as the volume integral up to those scales, $i$. e., integrated on a asymptotic surface $S(\lambda)$ with $\varrho \simeq \lambda^{-1}$. This ensures that the volume term in the definition of the Komar mass:

$$
2 \int_{\Sigma} \xi^{e} R_{e}^{d} \epsilon_{a_{1} \ldots a_{n-1} d}
$$

is finite even in the $\lambda \rightarrow 0$ limit.

Inspecting the formula for the variation of the total mass (4.4) written in terms of the "nhe" geometry,

$$
\delta M=-\frac{\lambda^{2}}{8 \pi} \int_{S(\lambda)} \varepsilon_{a_{1} \ldots a_{n-2} b c} \xi^{b} \nabla_{d}\left(h^{c d}-g^{c d} h\right),
$$

one sees that the leading order terms in $\varrho$ stems from the multiplicative terms involving the variation of the metric and the Christoffel symbols of (4.7): Following the condition that the variation should be finite in the $\lambda \rightarrow 0$ limit, one arrives at the boundary conditions for the near horizon metric as (4.8). Therefore, even changing dramatically the boundary conditions of the near horizon metric, these metric variations still yield sensible asymptotic charges in the full Kerr geometry. One should also remark that, from the discussion in the last Section, the fact that the near-horizon geometry is still $\mathrm{AdS}_{2} \times X$ after the metric variation means that 
one is keeping extremality. Thus one does not need to enforme similar constraints on the variation of the angular momenta.

To summarize, the boundary conditions (4.8) stem from the physical requirement that changes on the near extremal metric still allow for finite changes in the asymptotic charges of the full metric. As stated, the purpose of those boundary conditions is to provide the requirements on the space-times which will be counted by the asymptotic symmetries

$$
\xi_{\epsilon}=\epsilon(\phi) \partial_{\phi}-\varrho \epsilon^{\prime}(\phi) \partial_{\varrho}
$$

The surprising feature of such solution is that, despite its simplicity, it manages to be a orbit on the space of global gauge transformations, $i$. e., each variation counts exactly one diffeomorphically inequivalent metric. Without a microscopic theory, one cannot prove it so at this point. We hope to return to this point in the future.

\section{Central Charges}

In this section, we will describe the procedure to obtain asymptotic charges algebra with respect to the boundary conditions obtained in previous section. This algebra will be a conformal algebra with a central charge on which we will focus. As stated in the preceeding sections, the central charge is the key ingredient on character formulas for the number of states at a given level. The entropy obtained in this manner is, surprinsingly, equal to Bekenstein-Hawking entropy for an extremal Kerr black hole.

Most of this Section will follow the guidelines of [5], and, since the general form of the metric (3.9) poses no additional challenge, will be somewhat schematical. By the prescription (4.9) for the relation between Kerr mass and a divergent "nhe" mass, we have obtained the boundary conditions (4.8). These conditions allow one to obtain the the generators of the asymptotic symmetries

$$
\begin{aligned}
\xi_{\epsilon} & =\epsilon(\phi) \partial_{\phi}-\varrho \epsilon^{\prime}(\phi) \partial_{\varrho} \\
\zeta_{t} & =\partial_{t} .
\end{aligned}
$$

Observe that $\zeta_{t}$ generates time translations. Furthermore, since we are treating $\phi$ as a periodic, we may set $\epsilon_{n}(\phi)=-e^{-i n \phi}$, for $n$ an integer. Therefore, we have an infinite number of generators for the asymptotic symmetry generated by $\xi_{\epsilon}$, i.e., 
$\xi_{n}=\xi\left(\epsilon_{n}\right)$. These infinite generators are closed into a Diff $\mathrm{S}^{1}$ algebra:

$$
i\left[\xi_{n}, \xi_{m}\right]=(m-n) \xi_{m+n}
$$

For $n=0, \xi_{0}$ generates a simple rotational isometry, i.e., $U(1)$.

The next step is to obtain asymptotic charges associated with asymptotic symmetries, and then a Dirac algebra for these asymptotic charges. Conserved asymptotic charges associated with asymptotic symmetries $\zeta$ are defined by [19]:

$$
Q_{\zeta}[\bar{g}]=\frac{1}{8 \pi} \int_{S} k_{\zeta}[h ; \bar{g}],
$$

where $S=\partial \Sigma$ is the boundary of a codimension one hypersurface, and $h_{a b}$ encodes boundary conditions for the field (background) $\bar{g}_{a b}$. Furthemore, $k_{\zeta}$ is an asymptotically conserved $n-2$-form given by $k_{\zeta}[h ; \bar{g}]=k_{\zeta}^{[a b]}[h ; \bar{g}]\left(d^{(n-2)} x\right)_{a b}$, such that

$$
k_{\zeta}^{[a b]}[h ; \bar{g}]=\frac{\sqrt{-\bar{g}}}{16 \pi}\left(\zeta_{c} \bar{\nabla}_{\sigma} H^{c d a b}+\frac{1}{2} H^{c d a b} \partial_{c} \zeta_{d}\right)
$$

where the covariant derivative $\bar{\nabla}$ is with respect to background $\bar{g}_{a b}$, and $H^{c d a b}$ is a background tensor with indices symmetries similar to a Riemman tensor ${ }^{2}$

$$
\begin{aligned}
H^{c d a b}[h ; \bar{g}] & =-\hat{h}^{c b} \bar{g}^{d a}-\hat{h}^{d a} \bar{g}^{c b}+\hat{h}^{c a} \bar{g}^{d b}+\hat{h}^{d b} \bar{g}^{a c} \\
\hat{h}_{a b} & =h_{a b}-\frac{1}{2} \bar{g}_{a b} h .
\end{aligned}
$$

Obviously, in the above expression indices are raised and lowered with the background metric $\bar{g}_{a b}$.

The Dirac bracket algebra for these asymptotic charge may display a central charge. Formulae for this algebra and the central charges are

$$
\left\{Q_{\zeta_{1}}, Q_{\zeta_{2}}\right\}=\delta_{\zeta_{1}} Q_{\zeta_{2}}+K_{\zeta_{1}, \zeta_{2}}
$$

and

$$
K_{\zeta_{1}, \zeta_{2}}=\frac{1}{8 \pi} \int_{S} k_{\zeta_{1}}\left[\mathcal{L}_{\zeta_{2}} \bar{g}, \bar{g}\right]
$$

where $\mathcal{L}_{\zeta_{2}}$ is a Lie derivative along $\zeta_{2}$. We draw attention to the fact [19] that $K_{\zeta_{1}, \zeta_{2}}=-K_{\zeta_{2}, \zeta_{1}}$. We now use the above formalism for the asymptotic symmetries given (5.1) and (5.2). For the time translation $\zeta_{t}$, as discussed in [5], its associated

\footnotetext{
${ }^{2}$ See eqs. (6.19) and (6.20) in [19], which we reproduce here.
} 
asymptotic charge has to vanish identically. Therefore, this implies further supplementary boundary conditions. Now, the boundary conditions (4.8) does satisfy $Q_{\zeta_{t}} \equiv 0^{3}$.

For asymptotic charges associated to generators of the algebra (5.1), one obtain a Virasoro algebra with central charge computed from the integral (5.9). If we define Virasoro generators $L_{n}$ as $L_{n}=Q_{\xi_{n}}+\frac{3 J}{2} \delta_{n, 0}$. The by Dirac's quantization procedure $(\{\cdot, \cdot\} \rightarrow-i[\cdot, \cdot])$, then

$$
\left[L_{m}, L_{n}\right]=(m-n) L_{m+n}+\frac{c_{L}}{12} m\left(m^{2}-1\right) \delta_{m+n, 0},
$$

with

$$
c_{L}=12 J
$$

\subsection{Frolov Temperature}

The Frolov-Thorne temperature for Kerr black hole is obtained analogously as the Hawking temperature for Schwarzschild black hole. There, one first define a HartleHawking vacuum outside the black hole to be a mixed state $\rho=e^{-E / T_{H}}$. For the extremal Kerr back hole, this definition needs to be worked on since $T_{H}=0$. The solution for the mixed state stems from the existence of another thermodynamic potential related to the angular momentum $J$ of the black hole. Thus, from the first Law,

$$
T_{H} \delta S=\delta M-\Omega_{H} \delta J .
$$

In the extremal case, $T_{H} \delta S=0$, then $\delta M=\Omega_{H} \delta J$. Following [25], one has in this case that the entropy variation is given by $\delta S=\delta J / T_{L}$. Now, the entropy for the extremal Kerr black Hole is $S=2 \pi J$. Therefore we set the Frolov-Thorne temperature to be $T_{L}=1 / 2 \pi$. One notes at this point that this is the inverse of the periodicity of the coordinate $\phi$ defined at (3.8). In this fashion, it arises naturally as the periodicity required so that the metric has no conical singularity after analytic continuation to Euclidian time.

In the spirit of Section 4, where we have obtained boundary conditions (4.8), we may obtain the same value for $T_{L}$ by a semi-classical argument. The first step is to define an analogue of Hartle-Hawking vacuum for the Kerr Black Hole, i.e., Frolov-Thorne vacuum. For that, one consider, for simplicity, a scalar quantum field $\Phi$ in a classical four dimensional Kerr background. Now, expand this scalar field in

\footnotetext{
${ }^{3}$ see footnote 10 in [5].
} 
eigenstates of asymptotic energy $\omega$ and angular momentum $l, m$ as

$$
\Phi(x)=\sum_{\omega, l, m} \Phi_{\omega, l, m} e^{-i \omega \bar{t}+i m \bar{\phi}} F_{l}\left(\rho, x^{i}\right) .
$$

One now has to trace out the interior of the black hole to obtain the vacuum as a density matrix in the above basis as $e^{-\frac{\omega-\Omega_{H} m}{T_{H}}}$. However, since $T_{H}=0$, this expression for density matrix is not suitable. One way of solving of this problem is to rewrite the coordinates in the scaled form (3.8), and take the limit $\lambda \rightarrow 0$ after the procedure. In the coordinates (3.8), the field is written as

$$
\Phi(x)=\sum_{\omega, l, m} \Phi_{\omega, l, m} e^{-i \omega \frac{t}{\lambda}+i m\left(\phi-\Omega_{H} \frac{t}{\lambda}\right)} F_{l}\left(\lambda r, x^{i}\right),
$$

which can be suitably recast as

$$
\Phi(x)=\sum_{\omega, l, m} \Phi_{\omega, l, m} e^{-i\left(E_{R} t+J \phi\right)} F_{l}\left(\lambda r, x^{i}\right),
$$

with

$$
J \equiv m, \quad E_{R} \equiv \frac{\omega-\Omega_{H}}{\lambda} .
$$

Once again, one trace out the interior of the black hole. In this case, we obtain

$$
\rho=e^{-\frac{E_{R}}{T_{R}}-\frac{J}{T_{L}}}
$$

with

$$
T_{L}=\frac{\Omega_{H}}{2 \pi \omega} \quad \text { and } \quad T_{R}=\kappa
$$

in units of $M$.

Now, we take the limit $\lambda \rightarrow 0$. If $E_{R}$ is not zero, then the extremality limit $\kappa \rightarrow 0$ would make the density matrix (5.17) vanish. Then there would only be pure states. If one, on the other hand, enforces the extremal limit in terms of the double limit $\lambda \rightarrow 0$ and $\omega-\Omega_{H} \rightarrow 0$, then $E_{R}$ still tends to zero, but the density matrix is now non-vanishing, since

$$
T_{L}=\frac{1}{2 \pi}
$$

\subsection{Entropy}

The Bekenstein-Hawking entropy for Kerr black hole is obtained plugging the central 
charge (5.11) into Cardy-Verlinde formula

$$
S_{\text {micro }}=\frac{\pi^{2}}{3} c_{L} T_{L} .
$$

If we use the Frolov-Thorne temperatute for extremal Kerr black hole (2.4), then

$$
S_{\text {micro }}=2 \pi J \equiv S_{\text {Kerr }} .
$$

Therefore, via the counting of microstates encoded in central charge, $c_{L}$, we obtained a thermodynamic entropy $S_{\text {Kerr }}$.

We should however observe that use of Cardy-Verlinde formula here has to be done cum grana salis. Indeed, Cardy-Verlinde formula is valid, provided the theory is unitary and modular invariant, when $T \gg c$. But here $T_{L} \ll c_{l}$, or at best of the same order. The same happens in many other situations, e. g., in StromingerVafa work. There are some attempts to understand this numerical coincidence in the literature, still lacking a good explanation. This example of decoupling limit between the near horizon degrees of freedom is unique in the fact that it does not seem to rely on the usual theorems of nonrenormalizability of supersymmetric theories. It would be then interesting to explicit the exact property that protects the sector of the dual theory in the renormalization group flow.

\section{Conclusions}

In this letter we studied the underlying space-time mechanism behind the Kerr/CFT correspondence. We took an space-time approach, trying to understand the emergence of the conformal symmetry and to elucidate the mechanism behind the coincidence of Cardy's formula and the Bekenstein-Hawking entropy. We revisited, for the first part, that the conformal symmetry is a direct consequence of the existence of a zero-temperature Killing horizon. Heuristically, any such horizon will have $\partial / \partial \rho$ have its norm diverging as (3.4), and the $\mathrm{SL}(2, \mathbb{R})$ structure will appear.

For the second part, we have given some arguments that the Bekenstein-Hawking

entropy is again counting inequivalent metrics. The Kerr/CFT correspondence helps us count metrics which change global charges despite being generated by diffeomorphisms. When one restricts such metrics to the near horizon limit, one arrives at the boundary conditions proposed by [5]. Again heuristically, the boundary conditions are such that allows for finite displacements of the global charges of the entire black hole, even though the near horizon strutucture changes considerably. We then review 
some features of these global diffeomorphisms, which displaying a central charge in their representation in a extremal black hole background.

We conclude posing the question of what feature of the horizon is necessary for the statement that the sector of the dual theory is decoupling. This idea has been around some time under the name of "attractor mechanism" [26] (see also [27], and [28] for a discussion of the purported dual CFT). Albeit full supersymmetry seems unnecessary, the algebraic structure of the black hole like the existence of a twice repeated null principal vector should play a role. One should remember at this point that the existence of special algebraic structures is sufficient to prove that the spacetime is an exact target space for string theory, or, more generically, that the spacetime is free from quantum corrections to the geometry. The existence of a covariantly constant null vector field, for instance, falls into both categories $[29,30]$. We hope to say more along those lines in the near future.

\section{Acknowledgements}

We would like to thank Mirjam Cvetič, Brian Wecht and especially Luciano Barosi

for comments and suggestions. We would also like to thank Francisco Brito, Álvaro Ferraz for support. BCdC thanks the ICCMP-UnB, where part of this work was conducted. ARQ acknowledges partial support of FINATEC and also CNPq under grant no. 307760/2009-0.

\section{References}

[1] V. I. Arnold, Mathematical Methods of Classical Mechanics. Springer-Verlag, New York, 1989.

[2] J. D. Brown and M. Henneaux, Central Charges in the Canonical Realization of Asymptotic Symmetries: An Example from Three-Dimensional Gravity, Commun. Math. Phys. 104 (1986) 207-226.

[3] J. L. Cardy, Operator Content of Two-Dimensional Conformally Invariant Theories, Nucl. Phys. B270 (1986) 186-204.

[4] A. Strominger and C. Vafa, Microscopic Origin of the Bekenstein-Hawking Entropy, Phys. Lett. B379 (1996) 99-104, hep-th/9601029.

[5] M. Guica, T. Hartman, W. Song, and A. Strominger, The Kerr/CFT Correspondence, arXiv:0809.4266. 
[6] J. M. Bardeen and G. T. Horowitz, The extreme Kerr throat geometry: A vacuum analog of AdS(2) x S(2), Phys. Rev. D60 (1999) 104030, hep-th/9905099.

[7] V. P. Frolov and K. S. Thorne, Renormalized Stress-Energy Tensor Near the Horizon of a Slowly Evolving, Rotating Black Hole, Phys. Rev. D39 (1989) 2125-2154.

[8] A. M. Ghezelbash, Kerr-Bolt Spacetimes and Kerr/CFT Correspondence, arXiv:0902.4662.

[9] A. M. Ghezelbash, Kerr/CFT Correspondence in the Low Energy Limit of Heterotic String Theory, arXiv:0901.1670.

[10] T. Azeyanagi, N. Ogawa, and S. Terashima, Holographic Duals of Kaluza-Klein Black Holes, JHEP 04 (Nov., 2009) 061, arXiv:0811.4177.

[11] T. Azeyanagi, N. Ogawa, and S. Terashima, The Kerr/CFT Correspondence and String Theory, arXiv:0812.4883.

[12] H. Isono, T.-S. Tai, and W.-Y. Wen, Kerr/CFT correspondence and five-dimensional BMPV black holes, arXiv:0812.4440.

[13] D. D. K. Chow, M. Cvetic, H. Lu, and C. N. Pope, Extremal black hole/cft correspondence in (gauged) supergravities, arXiv:0812.2918.

[14] H. Lu, J. Mei, and C. N. Pope, Kerr-AdS/CFT Correspondence in Diverse Dimensions, JHEP 0904:054,2009 (JHEP 0904:054,2009) arXiv:0811.2225.

[15] C. Krishnan and K. Stanislav, A Comment on Kerr-CFT and Wald Entropy, Phys. Lett., B677 (2009) 326-331, arXiv:0903.2169.

[16] Y. Matsuo, T. Tsukioka, and C.-M. Yoo, Another Realization of Kerr/CFT Correspondence, arXiv:0907.0303.

[17] Y. Matsuo, T. Tsukioka, and C.-M. Yoo, Yet Another Realization of Kerr/CFT Correspondence, arXiv:0907.4272.

[18] J. Rasmussen, Isometry-preserving boundary conditions in the Kerr/CFT correspondence, arXiv:0908.0184.

[19] G. Barnich and F. Brandt, Covariant theory of asymptotic symmetries, conservation laws and central charges, Nucl. Phys. B633 (2002) 3-82, hep-th/0111246].

[20] J. Bardeen and R. Wagoner, Relativistic Disks. I. Uniform Rotation, Astrophys. J. 167 (1971) 359.

[21] R. M. Wald, General Relativity. The University of Chicago Press, 1984. 
[22] B. Carter, Black Holes Equilibrium States, in Black Holes (C. DeWitt and B. DeWitt, eds.). Gordon \& Breach, New York, 1973.

[23] J. M. Bardeen, B. Carter, and S. W. Hawking, The Four laws of black hole mechanics, Commun. Math. Phys. 31 (1973) 161-170.

[24] M. Bañados, Three-dimensional quantum geometry and black holes, hep-th/9901148.

[25] T. Hartman, K. Murata, T. Nishioka, and A. Strominger, CFT Duals for Extreme Black Holes, JHEP 04 (Nov., 2009) 019, arXiv:0811.4393.

[26] A. Sen, Black Hole Entropy Function, Attractors and Precision Counting of Microstates, Gen. Rel. Grav. 40 (Aug., 2008) 2249-2431, arXiv:0708.1270.

[27] D. Astefanesei, K. Goldstein, R. P. Jena, A. Sen and S. P. Trivedi, Rotating Attractors, JHEP 10 (2006) 058, hep-th/0606244.

[28] D. Astefanesei and K. Srivastava, CFT Duals for Attractor Horizons, Nucl. Phys. B822 (2009) 283-300, arXiv:0902.4033.

[29] A. A. Tseytlin, String vacuum backgrounds with covariantly constant null Killing vector and 2-d quantum gravity, Nucl. Phys. B390 (1993) 153-172, hep-th/9209023.

[30] A. A. Coley, G. W. Gibbons, S. Hervik, and C. N. Pope, Metrics With Vanishing Quantum Corrections, Class. Quant. Grav. 25 (2008) 145017, arXiv:0803.2438. 